\title{
AUTOMORPHISM GROUPS OF LOCALLY COMPACT REDUCTIVE GROUPS
}

\author{
T. S. WU \\ (Communicated by David G. Ebin) \\ Dedicated to Mr. Chu, Ming-Lun on his seventieth birthday
}

\begin{abstract}
A topological group $G$ is reductive if every continuous finite dimensional $G$-module is semi-simple. We study the structure of those locally compact reductive groups which are the extension of their identity components by compact groups. We then study the automorphism groups of such groups in connection with the groups of inner automorphisms. Proposition. Let $G$ be a locally compact reductive group such that $G / G_{0}$ is compact. Then $I\left(G_{0}\right)$ is dense in $A_{0}(G)$.
\end{abstract}

Let $G$ be a locally compact topological group. Let $A(G)$ be the group of all bi-continuous automorphisms of $G$. Then $A(G)$ has the natural topology (the so-called Birkhoff topology or $g$-topology $[1,3,4]$ ), so that it becomes a topological group. We shall always adopt such topology in the following discussion. When $G$ is compact, it is well known that the identity component $A_{0}(G)$ of $A(G)$ is the group of all inner automorphisms induced by elements from the identity component $G_{0}$ of $G$, i.e., $A_{0}(G)=I\left(G_{0}\right)$. This fact is very useful in the study of the structure of locally compact groups. On the other hand, it is also well known that when $G$ is a semi-simple Lie group with finitely many connected components $A_{0}(G)=I\left(G_{0}\right)$. The latter fact had been generalized to more general groups ([3]). Call a connected locally compact group a semisimple group if it does not contain any non-trivial connected abelian normal subgroups. Put in another way, a locally compact connected group is semisimple if and only if its radical is trivial. It is proved in [3] if $G$ is semi-simple then $A_{0}(G)=I(G)$. In fact, compact groups and connected semi-simple groups can both be put into one larger class of locally compact groups. Let $G$ be a locally compact topological group. We say that $G$ is reductive if and only if every continuous finite-dimensional linear representation of $G$ is semi-simple. Then compact groups and semi-simple groups both are reductive groups. We hastily remark that in our definition of reductive groups, we do not demand that the group has a faithful linear representation. Without such requirement, even in the case of analytic groups, the Levi factor may have non-finite centers

Received by the editors November 27, 1985 and, in revised form, September 3, 1986.

1980 Mathematics Subject Classification (1985 Revision). Primary 22D05. 
and also it may not be closed. This certainly causes some complications. On the other hand, the reductivity does impose quite strong structural conditions on the groups. In this note, we shall first study the structure of reductive groups. Then, we shall investigate the identity component of the group of automorphisms. It turns out that when $G$ is a compactly generated Lie group $A_{0}(G)=I\left(G_{0}\right)$. However, for general reductive groups, we do not have such strong results. We show that $I\left(G_{0}\right)$ is dense in $A_{0}(G)$. We shall give an example which shows that in general $A_{0}(G)$ is larger than $I\left(G_{0}\right)$.

The study of $A_{0}(G)$ is not new ([7]). In [7], it was stated that $A_{0}(G)=$ $I\left(G_{0}\right)$. However, the discussion relies on a false statement from [2]. Also some preliminary discussion and results are false. Our approach here is somewhat different from [7].

\section{REDUCTIVE LIE GROUPS}

Let $G$ be a reductive Lie group. By definition, every continuous finite$\operatorname{dim} G$-module is semi-simple. This condition is equivalent to the condition $H^{1}(G, V)=0$ for every finite-dim continuous $G$-module $V$. (For a proof of this fact, see Mostow [10, 2.1.2].) When $G$ is also compactly generated, by a result of Garland and Goto [11, Proposition 4], $A_{0}(G)=I\left(G_{0}\right)$. So, we have the following statement.

(1.1) Proposition. Let $G$ be a compactly generated reductive Lie group. Then $A_{0}(G)=I\left(G_{0}\right)$.

(1.2) Proposition. Let $G$ be a reductive Lie group. Let $G_{0}$ be the identity component. Then the radical $R$ of $G$ is an abelian group and the Levi factor $S$ of $G_{0}$ is a normal subgroup.

Proof. Considering the adjoint representation of $G$ on the Lie algebra $L(G)$, the results follow immediately from the reductivity of $G$.

(1.3) Remark. We do not assume that $G$ has a faithful finite-dim linear representation, so $S$ may not be closed in $G$; also it may have a non-finite center (see examples in section 3 ).

\section{LOCALLY COMPACT REDUCTIVE GROUPS}

In this section, $G$ denotes a locally compact Hausdorff reductive group with compact component group, i.e., $G / G_{0}$ is compact. $G$ is a $C$-group ([5]). It has a maximal compact normal subgroup $K$. Let $G_{1}=K G_{0}$. Then $G_{1}$ is an open normal (characteristic) subgroup of $G$. Because $G / G_{1}$ is finite, $G_{1}$ is reductive. Let $Z_{G_{1}}(K)$ be the centralizer of $K$ in $G_{1}$. Then $G_{1}=K Z_{G_{1}}(K)$ ([5]). Let $F$ be the identity component of $Z_{G_{1}}(K), G_{1} / K=K G_{0} / K$ is connected so $K Z_{G_{1}}(K) / K$ is connected. Since $Z_{G_{1}}(K) / K \cap Z_{G_{1}}(K)$ is isomorphic with $K Z_{G_{1}}(K) / K$, it is also connected. $K \cap Z_{G_{1}}(K)$ is compact. Hence $\left(K \cap Z_{G_{1}}(K)\right) F$ is a closed subgroup of $Z_{G_{1}}(K)$. But $Z_{G_{1}}(K) / F$ is totally disconnected. This shows that $\left(K \cap Z_{G_{1}}(K)\right) F=Z_{G_{1}}(K)$. Therefore, $K F=Z_{G_{1}}(K) K=G_{1}$. 
(2.1) Proposition. $F$ is a reductive group. Let $R$ be the radical of $F$. Then we have the following conditions:

(1) $R$ is central in $G_{1}$.

(2) There is a subgroup $S$ which is a continuous isomorphic image of an analytic semi-simple group $S^{*}$ such that $F=R \bar{S}$ and $S^{*}$ has no non-trivial compact factor.

(3) $F / \bar{S} \approx R / R \cap \bar{S}$ is compact.

All the subgroups mentioned above are characteristic subgroups of $G$. Finally, if $G$ has all the above properties, then $G$ is reductive.

Proof. $G$ can be approximated by reductive Lie groups. By Proposition 1.2, the radical $R(G)$ of $G$ is an abelian group. $R(G) \subset G_{0}$, and is central in $G_{0}$.

Let $E=F \cap K . E$ is a compact central subgroup of $K$ and $F$, so it is central in $G_{1}=K F$. Now, $F / F \cap K$ is reductive, and $F \cap K=E$ is reductive. By a theorem attributed to Chevally ([8, Theorem 3.2]), $F$ is reductive. $F$ is a connected locally compact group. Locally, we have the neighborhood $E \times L$, where $L$ is a local Lie group. $L$ generates an analytic subgroup $L$, i.e., $L=$ $\bigcup_{n=1}^{\infty} L^{n}$. Let $S$ be a semi-simple factor. Because $F$ does not contain any compact semi-simple subgroup, $S$ is a semi-simple subgroup without compact factor. The radical $R$ of $F$, as noted above, is central in $F$. This implies $S$ is characteristic in $F$, a fortiori, in $G$. The radical $R$ of $F$ is abelian, $R=V \times Q, V$ a vector group and $Q$ a compact connected abelian group, $Q \subset E, E / Q$ is a compact totally disconnected subgroup. There exists a compact totally disconnected subgroup $M$ such that $E=Q M$ ([6]). So $F=$ $E V S=M Q V S=M R S$. Because we do not assume that $G$ has a faithful linear representation, $S$ may not have finite center; also it may not be closed in $G$. Observe that $F / \bar{S}=M R S / \bar{S}$ is connected, so $M R / M R \cap \bar{S}$ is connected. Then $M / M \cap(M R \cap \bar{S})$ is connected. But $M$ is totally disconnected. From this, we know $M \cap \bar{S}=M, M \subset \bar{S}$. Therefore $F=R S=V Q \bar{S}$. Because $F$ is reductive, the homomorphic image of $F$ must be also reductive. $F / \bar{S}=$ $R / R \cap \bar{S}$ is reductive. We conclude that $R \cap \bar{S}$ must be a uniform subgroup of $R . R$ is central in $F, F$ centralizes $K$. Hence $R$ is central in $G_{1}$. The converse of the theorem is clear. We skip the proof.

(2.2) Lemma. $A_{0}(\bar{S})=I(\bar{S})$.

Proof. $S$ is a characteristic subgroup of $S$, so for every $\alpha$ in $A(\bar{S}), \alpha(S)=$ $S$. The differential $d \alpha$ of $\alpha$ induces an automorphism of the Lie algebra $L(S)$. Because $A_{0}(S)$ is connected, the image of $A_{0}(S)$ in the automorphism group of the semi-simple Lie algebra $L(S)$ is contained in the group of inner automorphisms. On the other hand, the adjoint representation of $S$ on $L(S)$ is onto the group of inner automorphisms of $L(S)$. Therefore, for each $\alpha \in$ $A_{0}(S)$, there exists an $s$ in $S$ such that $d \alpha=d I_{s}, d I_{s}^{-1} \circ \alpha$ is the identity on $L(S)$. Thus $I_{s}^{-1} \circ \alpha$ is the identity on $S / Z(S)$. But $Z(S)$ is discrete; and $S$ 
is connected. This implies $I_{s}^{-1} \circ \alpha$ is trivial on $S$, i.e., $I_{s}=\alpha$ on $S$. Hence $\alpha=I_{s}$ on its closure $\bar{S}$, and $A_{0}(\bar{S})=I(\bar{S})$.

(2.3) Lemma. $A_{0}(F)=I_{F}(F)$.

Proof. As noted above, $F=V Q S=V E S$. The choice of $V$ is not unique. We choose one for the rest of the proof. Let $P_{\lambda}$ be any compact normal subgroup of $F$ such that $F / P_{\lambda}$ is a Lie group. $P_{\lambda} \subset E . E$ is a characteristic subgroup. It is invariant under $A_{0}(F)$. The restriction of $A_{0}(F)$ on $E$ is a connected subgroup of $A_{0}(E)$. But $E$ is abelian and compact. $A_{0}(E)=I\left(E_{0}\right)$ is trivial. Therefore, $A_{0}(E)$ acts trivially on $P_{\lambda}$ also. Because $F / P_{\lambda}$ is reductive $A_{0}\left(F / P_{\lambda}\right)=I\left(F / P_{\lambda}\right)$. Hence for each $\alpha \in A_{0}(F)$, the induced automorphisms by $\alpha$ on $F / P_{\lambda}$ is inner. But $V P_{\lambda} / P_{\lambda}$ is central in $F / P_{\lambda}$. Hence the induced automorphism is trivial on $V P_{\lambda} / P_{\lambda}$. This in turn means $\alpha(x)=x y_{\lambda}$ for each $x \in V$ and some $y_{\lambda}$ in $P_{\lambda}$. Now, let $P_{\lambda}$ approach the identity. We have $\alpha(x)=\lim x y_{\lambda}=x$. Hence $x$ is pointwise fixed for each $\alpha \in A_{0}(F)$.

Now, $S$ is invariant under $A_{0}(F)$. By Lemma 2.2, for each $\alpha \in A_{0}(F)$, there exists $s \in S$ such that $\alpha\left|S=I_{s}\right| S$. Because $V E$ is central in $F, a$ fortiori, $\alpha=I_{s}$ on $F$. The proof is now complete.

(2.4) Proposition. Let $G$ be a locally compact reductive group. Assume $G / G_{0}$ is compact. Let $K$ be the maximal compact normal subgroup of $G$. Then $G_{1}=K G_{0}$ is an open normal subgroup of $G$, and $A_{0}\left(G_{1}\right)=I_{G_{1}}\left(G_{0}\right)$.

Proof. $K$ is characteristic, so it is invariant under $A_{0}\left(G_{1}\right)$. Because $K$ is compact, given any $\alpha \in A_{0}\left(G_{1}\right)$, there exists $k \in K_{0} \subset G_{0}$ such that $\alpha \mid K=$ $I_{k} \mid K$. By Lemma 2.3, $\alpha\left|F=I_{r}\right| F$ for some $r \in S$. Recall that $F$ centralizes $K$. From this, we have that $\alpha=I_{k} I_{r}=I_{r} I_{k}=I_{r k}$, with $r k \in G_{0}$. So $A_{0}\left(G_{1}\right)=I_{G_{1}}\left(G_{0}\right)$.

(2.5) Proposition. Let $G$ be a locally compact reductive group such that $G / G_{0}$ is compact. Then $I\left(G_{0}\right)$ is dense in $A_{0}(G)$.

Proof. Let $K$ be the maximal compact normal subgroup of $G$. For any compact normal subgroup $P$ of $G$ such that $G / P$ is a Lie group, $P \subset K . A_{0}(G)$ leaves $K$ invariant. Furthermore, the restriction of $A_{0}(G)$ to $K$ is inner. Hence $A_{0}(G)$ leaves $P$ invariant. Therefore, for each $\alpha \in A_{0}\left(G_{1}\right), \alpha$ induces an automorphism on $G / P$. Because $G / P$ is reductive and compactly generated, by Proposition 1.1, the induced automorphism is inner. This implies for each $\alpha \in A_{0}\left(G_{1}\right)$, there exists an element $g \in G_{0}$ such that $\alpha(x P)=I g(x P)$, or $I_{g}^{-1} \circ \alpha(x P)=x P$. Now, the typical neighborhood for the identity map of $A(G)$ is of the form $[Y, V]$, where $Y$ is a compact subset of $G, V$ a neighborhood of the identity $e$ of $G$, and $[Y, V]=\left\{\alpha \in A(G), \alpha(g) g^{-1} \in V\right.$, $\alpha^{-1}(g) g^{-1} \in V$ for all $\left.g \in Y\right\}$. Now, given any $V$, neighborhood of $e$, choose any $P$ such that $P \subset V$, and $G / P$ is Lie. Then, from the above discussion, given any $\alpha$ in $A_{0}(G)$, there exists $g \in G_{0}, I_{g}^{-1} \circ \alpha \in[Y, V]$. Hence $I\left(G_{0}\right)$ is dense in $A_{0}(G)$. 


\section{EXAMPLES}

In this section, we shall give several simple examples to illustrate the contents of previous sections.

(3.1) Non-closed analytic semi-simple subgroups. Let $S^{*}$ be any analytic semisingle group with infinite center, say $Z\left(S^{*}\right)$ is isomorphic with integer group $Z$. Let $A$ be any compact monothetic group with generator $U$. Let $G^{*}=$ $A \times S^{*}$. Let $D \equiv\left\{\left(U^{n}, n\right): n\right.$ is any integer $\}$. Then $D$ is a closed central subgroup of $G^{*}$. Let $G$ be the quotient group $G^{*} / D$ and $\Pi$ be the canonical homomorphism from $G^{*}$ onto $G$. Then $\Pi\left(S^{*}\right)$ is an analytic subgroup of $G$, whose closure is $G$.

If we had chosen $A$ to be a Lie group, then $G$ is also a Lie group. This is so because $D$ is discrete, and $G^{*}$ is a covering group of $G$.

We remark that when $A$ is a Lie group, $G=\Pi\left(A_{0}\right) \Pi\left(S^{*}\right)$. On the other hand, when $A$ is not a Lie group and $A$ is not connected, then $G=\Pi(A) \Pi\left(S^{*}\right) \supset$ $\Pi\left(A_{0}\right) \Pi\left(S^{*}\right)$. The last containment is proper.

(3.2) Non-compact subgroup $A$. Let $S^{*}$ be an analytic semi-simple group with its center isomorphic with $\mathbf{Z} \times \mathbf{Z}$. For instance, we can take $S L(2, \mathbf{R})^{\sim} \times$ $S L(2, \mathbf{R})^{\sim}$, here $S L(2, \mathbf{R})^{\sim}$ is the universal covering group of $S L(2, \mathbf{R})$. Let $A \cong \mathbf{R}$, the additive group of real numbers. Pick any two rationally independent numbers $\alpha$ and $\beta$. Form $G^{*}=R \times S^{*}$. Let $D=\{(m \alpha+n \alpha, m, n): m, n$ integers $\} \subset R \times \mathbf{Z} \times \mathbf{Z} \subset R \times S^{*}$. Then $G=G^{*} / D$ has $\Pi\left(S^{*}\right)$ as a dense subgroup.

(3.3) $I\left(G_{0}\right)$ is a proper subgroup of $A_{0}(G)$. Let $S_{i}^{*}$ be a copy of an analytic semi-simple group with center $Z$ isomorphic with integers, $i=1,2$. Let $S^{*}=S_{1}^{*} \times S_{2}^{*} \supset \mathbf{Z} \times \mathbf{Z}$. Let $\rho_{s}$ be the automorphism of $S^{*}$ defined by $\rho_{s}(x, y)=(y, x)$. Let $A$ be a compact totally disconnected monothetic group with generator $U$. Then we have the automorphism $\rho_{A}$ of $A$ defined by $\rho_{A}(a)=a^{-1}$. Then we have the automorphism $\rho^{*}$ on $A \times S^{*}, \rho^{*}(a, x, y)=$ $\left(a^{-1}, y, x\right)$. We can form the semi-direct product $\left(A \times S^{*}\right) \times\left\{\right.$ id,$\left.\rho^{*}\right\}=G^{*}$. Let $D=\left\{U^{m}, m,-m\right) \mid m$ any integer $\}, D \subset A \times S^{*}$. We can identify $D$ as a subgroup of $G^{*}$. Then $D$ is a normal subgroup of $G^{*}$. Let $G=G^{*} / D$. Let $G_{1}$ be the image of $A \times S^{*}$. Then $G_{1}$ is an open subgroup of $G$, and it has index two in $G$. The coset other than $G_{1}$ in $G / G_{1}$ is the one containing $\Pi\left(\rho^{*}\right)$, where $\Pi$ is the canonical homomorphism $G^{*} \rightarrow G$. It is not difficult to see that $A_{0}\left(G_{1}\right)=I\left(G_{1}\right)$. Now, to see that $I\left(G_{0}\right)$ is a proper subgroup of $A_{0}(G)$, consider the inner automorphism $I(e, m, n, \mathrm{id})$ induced by the element $(e, m, n, \mathrm{id})$ which is from the center of $\Pi\left(S^{*}\right)$. Then $I(e, m, n, \mathrm{id})\left(\Pi\left(\rho^{*}\right)\right)=\Pi\left(e, m-n, n-m, \rho^{*}\right),(m, n) \in \mathbf{Z} \notin \mathbf{Z}$. When $m, n$ tend to $\infty$, we can choose various subsequences so that it converges to various points $\Pi\left(a, e, e, \rho^{*}\right), a \in A$. For any $a \in A$, define $\phi_{a}: G \rightarrow G$ by $\phi_{a} \mid G_{1}=$ identity map, $\phi_{a}\left(\Pi\left(\rho^{*}\right)\right)=\Pi(a)\left(\Pi\left(\rho^{*}\right)=\Pi\left(a \rho^{*}\right)\right.$. Then $\phi_{a} \in I\left(G_{0}\right)^{-}$. However not every $\phi_{a}$ is induced by an inner automorphism. In fact, in order for 
$\phi_{a}$ to be inner, it is necessary and sufficient that $a=b^{2}$ for some $b \in A$. If we have chosen $A$ to be the dyadic integers, then $U$ has no square root. Hence $\phi_{u}$ is not inner.

It is interesting to note in [3], the following theorem was proved: Let $G$ be a locally compact group such that $G_{0}$ is semi-simple and $G / Z_{G}\left(G_{0}\right)$ is connected. Then $A_{0}(G)=I\left(G_{0}\right)$. Here $Z_{G}\left(G_{0}\right)$ is the centralizer of $G_{0}$ in $G$. From the above example, we see that without the condition of $G / Z_{G}\left(G_{0}\right)$ being connected, the assertion may not be true.

\section{REFERENCES}

1. P. Chen and T. S. Wu, On the automorphism groups of locally compact groups, Tamkang Journal of Math. vol. 17, no. 2, (1986), 99-116.

2. M. Goto, Linear representations of topological groups, Proc. Amer. Math. Soc. 1 (1950), 161165 (MR \#3571).

3. S. Grosser, O. Loos and M. Moskowitz, Uber automorphism-engruppen lokal-Kompakter Gruppen und Derivationen. Von Lie-Gruppen, Math. Z. 114 (1970), 321-339 (MR 41 \#8575).

4. G. Hochschild, The structure of Lie groups, Holden Day, New York, 1965.

5. K. Iwasawa, On some types of topological groups, Ann. of Math. (2) 50 (1949), 507-558 (MR $10 \# 679$ ).

6. D. H. Lee, Supplements for the identity component in locally compact groups, Math. Z. 104 (1968), 28-49 (MR 36 \#6534).

7. __, Reductivity and automorphism groups of locally compact groups, Trans. Amer. Math. Soc. 221 (1976), 379-389.

8. G. Hochschild and G. D. Mostow, Representations and representative functions of Lie groups, Ann. of Math. (2) 65 (1957), 495-542.

9. G. D. Mostow, Cohomology of topological groups and solvmanifolds, Ann. of Math. (2) 73 (1961), 20-49 (MR 23 \#A2484).

10. __ Arithmetic subgroups of groups with radicals, Ann. of Math. (2) 93 (1971), 401-438.

11. H. Garland and M. Goto, Lattices and the adjoint group of a Lie groups. Trans. Amer. Math. Soc. 124 (1967), 450-460.

Department of Mathematics and Statistics, Case Western Reserve University, Cleveland, OHio 44106 